\title{
Analysis of the Spatial Properties of Correlated Photon in Collinear Phase-Matching
}

\author{
Lin Yin ${ }^{1,2}$, Jianjun $\mathrm{Li}^{1}$, Wenchao Zhai ${ }^{1}$, Maopeng Xia ${ }^{1}$, Youbo $\mathrm{Hu}^{1}$ and Xiaobing Zheng ${ }^{1, *}$ \\ 1 Key Laboratory of Optical Calibration and Characterization, Anhui Institute of Optics and Fine Mechanics, \\ HFIPS, Chinese Academy of Sciences, Hefei 230031, China; ylxw52@mail.ustc.edu.cn (L.Y.); \\ jjli@aiofm.ac.cn (J.L.); wczhai@aiofm.ac.cn (W.Z.); mpxia@aiofm.ac.cn (M.X.); youbohu@aiofm.ac.cn (Y.H.) \\ 2 Science Island Branch, Graduate School of USTC, Hefei 230026, China \\ * Correspondence: xbzheng@aiofm.ac.cn
}

check for updates

Citation: Yin, L.; Li, J.; Zhai, W.; Xia, M.; Hu, Y.; Zheng, X. Analysis of the Spatial Properties of Correlated Photon in Collinear Phase-Matching. Photonics 2021, 8, 12. https:// doi.org/10.3390/photonics 8010012

Received: 9 December 2020

Accepted: 4 January 2021

Published: 7 January 2021

Publisher's Note: MDPI stays neutral with regard to jurisdictional clai$\mathrm{ms}$ in published maps and institutional affiliations.

Copyright: $(\odot 2021$ by the authors. Licensee MDPI, Basel, Switzerland. This article is an open access article distributed under the terms and conditions of the Creative Commons Attribution (CC BY) license (https:// creativecommons.org/licenses/by/ $4.0 /)$.

\begin{abstract}
In this paper, the spatial properties of correlated photon in collinear phase-matching in the process of spontaneous parametric down conversion (SPDC) are researched. Based on the study of the phase-matching angle, non-collinear angle, and correlated photon wavelength, a theoretical model of non-collinear angular variation is derived, which can be used to estimate and predict the width of the correlated photon ring. The experimental measurement is carried out with CMOS camera, and the measurement results are consistent with the theoretical simulation results, which verifies the rationality of theoretical reasoning. Meanwhile, the change of the correlated photon divergence angle outside the crystal is studied, the closer the wavelength is to the degenerate, the smaller the measurement value of the divergence angle, which is agreement with the theoretical simulation. The results of the study play a reference role in the evaluation of the spatial properties of correlated photon and lay a foundation for the measurement of the correlated photon number rate and the calibration of a photodetector.
\end{abstract}

Keywords: spontaneous parametric down conversion; collinear phase-matching; non-collinear angular variation; divergence angle

\section{Introduction}

Spontaneous parametric down conversion (SPDC) is a nonlinear optical process in which a higher energy photon from laser beam splits into a pair of lower energy photons in the nonlinear crystal [1-5]. The photon pairs are produced simultaneously, but the properties of the individual photon are free and different, depending on the electric field and intensity of the higher energy photon during the down conversion process [6,7]. The two photons, called entangled photons or correlated photons [8], have a strict entanglement property in polarization time and space [9], which has been applied in quantum cryptography, quantum simulation, and quantum metrology [10-17]. In recent years, it is used for the absolute measurement of the quantum efficiency of photodetectors in the time-correlated single photon counting regime without relying on the calibrated standards [18-22]. During this process, the emission cone properties of correlated photons, such as spectral range, non-collinear angle, and divergence angle, play an important role in the selection of photosensitive surface of photodetector and the determination of its position. The SPDC of the down conversion process has two types depending on whether the correlated photons have the same or orthogonal polarization. If produced correlated photon pairs have the same polarization, but opposite to the pump, it is labelled as type-I down conversion, and vice versa, labelled as type-II down conversion [23-25]. Moreover, the pair of photons generated propagate in the same direction as the pumped photons, which is collinear phase-matching. Conversely, when the photon pairs travel in a different direction than the pump, it is the non-collinear phase-matching. The collinear phase-matching is a special case of non-collinear phase-matching. Compared with non-collinear phase-matching, 
the near-optical axis two-photon field of the collinear phase-matching can be carried out around the same propagation direction, and the spatial entanglement states are simple and easy to understand $[2,26,27]$. Also, the presence of Poynting vector walk-off effect will cause the spatial offset of the correlated photon increasing and reduce the conversion efficiency; for the collinear phase-matching, the spatial shape of the correlated photons due to the walk-off effect can be negligible $[27,28]$.

In the previous literature [29-33], spectral bandwidth and gain bandwidth models were constructed from crystal length, group velocity, and group velocity dispersion, etc. However, the model of the non-collinear angular variation, correlated photon ring width, and divergence angle is not perfect in terms of the construction and analysis of the system from the aspects of phase-matching angle, non-collinear angle, and phase mismatch. In this paper, we analyze the spatial properties of the emitted photons in collinear phase-matching via non-collinear angular variation and divergence angular spectrum. Under the condition of the degenerate wavelength collinear phase-matching, we first theoretically calculate the relationship between phase matching angle, non-collinear angle, and parametric wavelength, construct a model of non-collinear angular variation and ring width of correlated photon. Then, combined with the experimental measurement results, the correctness and rationality of the theoretical simulation results are proven. Moreover, combining the non-collinear angle with Snell's Law [34] to calculate the divergence angle outside the crystal at different wavelengths, and the experiment verifies the rationality of the theory. The properties and influence factors of SPDC process emission photons in uniaxial crystals with collinear phase-matching can be better informed. It lays a foundation for optimizing the SPDC process and carrying out correlated photon rate measurement and detector calibration.

\section{Theoretical Estimation of the Correlated Photon Ring Properties}

The SPDC process where the pump photon at energy $\hbar \omega_{p}$ splits into a correlated photon pair of signal and idler within the uniaxial crystal of $\mathrm{BBO}\left(\beta-\mathrm{BaB}_{2} \mathrm{O}_{4}\right.$ : barium metaborate) which energies are respectively $\hbar \omega_{s}$ and $\hbar \omega_{i}$, does follow energy conservation and momentum conservation rules so that [35]:

$$
\begin{gathered}
\hbar \omega_{p}=\hbar \omega_{s}+\hbar \omega_{i} \\
k_{p}=k_{s}+k_{i}
\end{gathered}
$$

where $\hbar$ is the Planck constant; $\omega_{j}$ and $k_{j}$ are the angular frequency and the wave vector for the pump, signal and idler modes $(j=p, s, i)$. The Equation (2) is also known as the phase-matching condition. In order to achieve the maximum conversion efficiency, Equations (1) and (2) need to be met simultaneously.

Figure 1 denotes the produced correlated photon ring profile due to a pump beam interacts with the $\mathrm{BBO}$ crystal. Equation (2) is rewritten as the wave vector models of pump and parametric (signal and idler):

$$
\begin{gathered}
k_{p}=k_{s} \cos \alpha+k_{i} \cos \beta \\
k_{s} \sin \alpha=k_{i} \sin \beta \\
k_{j}=\frac{2 \pi n_{j}}{\lambda_{j}}(j=p, s, i)
\end{gathered}
$$

where $\alpha$ and $\beta$ are the angles of signal and idler with pump beam, respectively. In addition to this the $\alpha$ (or $\beta$ ) is non-collinear angle in the crystal and it is called collinear phasematching as $\alpha=\beta=0^{\circ}$, which is a limit case of the non-collinear interaction scheme. The $n_{j}$ and $\lambda_{j}$ stand for the refraction index and wavelength, respectively. Thus, the relation of three wavelengths of signal (s), idler (i) and pump $(p): \frac{1}{\lambda_{p}}=\frac{1}{\lambda_{s}}+\frac{1}{\lambda_{i}}$ is satisfied. 


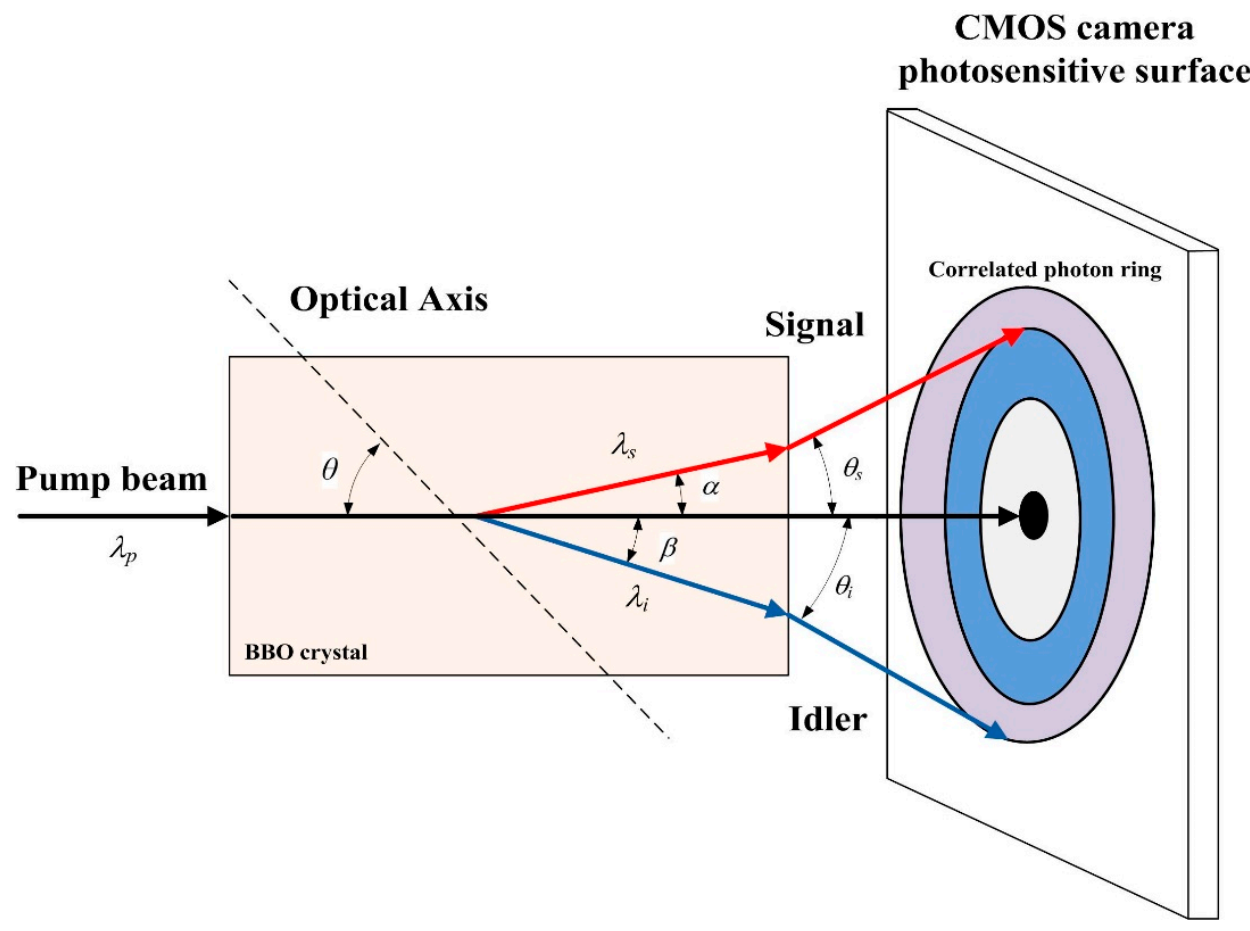

Figure 1. A pump beam interacts with the BBO crystal to produce a correlated photon ring, and the profile is recorded by the CMOS camera photosensitive surface.

In the paper, the SPDC process occurs for a pump wavelength of $266 \mathrm{~nm}$ and interacts with the nonlinear crystal. It is assumed that the generated parametric photons have a wavelength range of $400 \mathrm{~nm}-794 \mathrm{~nm}$, and the signal wavelength $\lambda_{s}$ is $400 \mathrm{~nm}-532 \mathrm{~nm}$. Then, the sum of the vector modes of parametric photons is $K_{\text {sum }}$ :

$$
K_{\text {sum }}=\frac{2 \pi}{\lambda_{s}}\left[n_{s}+\frac{n_{i}}{\lambda_{p}}\left(\lambda_{s}-\lambda_{p}\right)\right]
$$

where $n_{S}$ and $n_{i}$ are the refraction index of parametric photons.

For negative uniaxial BBO crystals with $n_{e}<n_{0}$, type I down conversion refers to the generated two-photon pairs with ordinary state, whose refractive index does not depend on the propagation direction. However, the pump beam as extraordinary state and the refractive index $n_{p}(\theta)$ associates with the propagation direction of light [36]:

$$
n_{p}(\theta)=\frac{n_{p}^{o} \cdot n_{p}^{e}}{\sqrt{\left(n_{p}^{o} \sin \theta\right)^{2}+\left(n_{p}^{e} \cos \theta\right)^{2}}}
$$

where $n_{p}{ }^{0}$ and $n_{p}{ }^{e}$ respectively represent the main refractive index of pump beam in the crystal, which can be calculated by the Sellmeier formulas of the BBO crystal $[37,38]$. The $\theta$ is the angle between the vector direction of extraordinary light (e light) and the optical axis of the crystal, also known as phase-matching angle.

The variation of $K_{\text {sum }}$ and $\theta$ at different signal wavelength is illustrated in Figure 2. It is clearly observed that the $K_{\text {sum }}$ is inversely proportional to $\lambda_{s}$, but $\theta$ is directly proportional to $\lambda_{s}$. That is, with the increase of the signal wavelength, the sum of the vector modes of parametric decreases while the phase-matching angle increases. As a result, when the maximum value $K_{\text {sum-max }}$ is $3.9735 \times 10^{7} \mathrm{~m}^{-1}$ at $400 \mathrm{~nm}$ and minimum value $K_{\text {sum-min }}$ is $3.9547 \times 10^{7} \mathrm{~m}^{-1}$ at $532 \mathrm{~nm}$. The pump vector mode is at minimum, $k_{p}(\theta)=K_{\text {sum-min }}$, according to Equations (6) and (7), the minimum value of refractive index $n_{p}(\theta)_{\min }$ and the maximum value of phase-matching angle $\theta_{\max }$ are 1.6742 and $47.6339^{\circ}$ respectively. Simi- 
larly, $k_{p}(\theta)=K_{\text {sum-max }}$, namely, the maximum refractive index $n_{p}(\theta)_{\max }$ and the minimum phase-matching angle $\theta_{\min }$ are 1.6822 and $44.4721^{\circ}$, respectively.

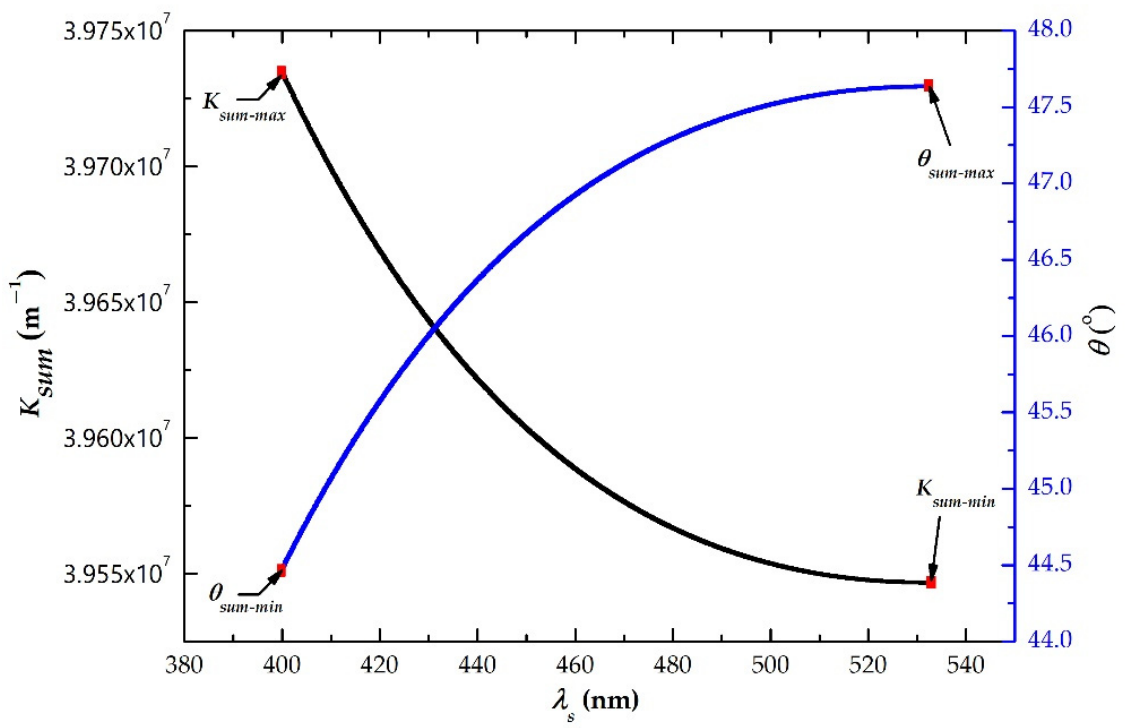

Figure 2. The dependence of the sum of the vector modes of parametric $K_{\text {sum }}$ and phase-matching angle $\theta$ on different signal wavelength $\lambda_{s}$.

As the phase-matching angle changes between $44.4721^{\circ}$ and $47.6339^{\circ}$, the wavelengths of signal and idle which output from the back of the crystal also changes, as shown in Figure 3. In the case of collinear phase-matching, the parametric wavelengths are $400 \mathrm{~nm}$ (for the signal photon) and $794 \mathrm{~nm}$ (for the idler photon) at $44.4721^{\circ}$. In order to satisfy the conservations of energy and momentum of the Equations (1) and (2), with the increase of phase-matching angle $\theta, \lambda_{s}$ increase while $\lambda_{i}$ decrease, all the way to $\theta$ is $47.6339^{\circ}, \lambda_{s}=\lambda_{i}=532 \mathrm{~nm}$ (for the degenerate photon pair). Of course, not only does the parametric photon pair with strict collinear phase-matching, but the satisfied non-collinear phase-matching condition can be emitted.

The key point of the correlated photon rate measurement and detector calibration using the SPDC is to determine the location and properties of the correlated photon ring. For this, it is important to concentrate on the study of the spatial distribution properties of the correlated photons within and outside the $\mathrm{BBO}$ crystal, which is the type I phasematching of the degenerate wavelength collinear $\left(\lambda_{s}=\lambda_{i}=2 \lambda_{p}=532 \mathrm{~nm}\right)$. When the pump beam interacts with the crystal surface at a certain angle, the correlated photons that satisfy the phase-matching requirements are produced, and the photons at different wavelengths correspond to different non-collinear angles, then, travel behind the crystal along different divergence angles. It is conical in space and ring-shaped on the photosensitive surface of the CMOS camera, which is perpendicular to the direction of the pump beam propagation. Moreover, the degenerate correlated photon pair is co-propagation with the pump, that is, $0^{\circ}$ of non-collinear angle, and the projection on the camera's photosensitive surface is beam-like, as shown in Figure 1.

From Equation (2), with the perfect phase-matching, the value of phase mismatch of three wave vectors (pump, signal and idler) in the crystal is 0 , expressed as $\Delta k=0$, and $\Delta k=k_{s}+k_{i}-k_{p}$. In practical applications, there are many factors such as pump beam dispersion, cutting angle deviation and crystal temperature instability, and $\Delta k \neq 0$, which reduces the efficiency of crystal conversion. In general, specifies the absolute value of the maximum phase mismatch mode allows $|\Delta k|=\frac{\pi}{L}$, where $L$ is the length of the interaction of three waves in the crystal $[29,39]$. From the previous analysis, it can be determined that in the process of SPDC, the phase-matching angle of the crystal meeting the conditions can emit correlated photons with a wide spectral range, as shown in Figure 3. 
The laser of $266 \mathrm{~nm}$ pumps the BBO crystal of phase-matching angle of $47.6339^{\circ}$, generated the correlated photons in $532 \mathrm{~nm}$ collinear while others noncollinear, and carries out a research of spatial distribution characteristics. Also, the spectral range emitted from the crystal is wide, however, the non-collinear angle variations of different wavelength correlated photons that satisfy the phase-matching criterial has a range, set as $\Delta \alpha$ (as signal) and $\Delta \beta$ (as idler). The smaller the value of $\Delta \alpha$ (or $\Delta \beta$ ), the narrower the correlated photon ring received on the CMOS camera's photosensitive surface, and the converse is wider.

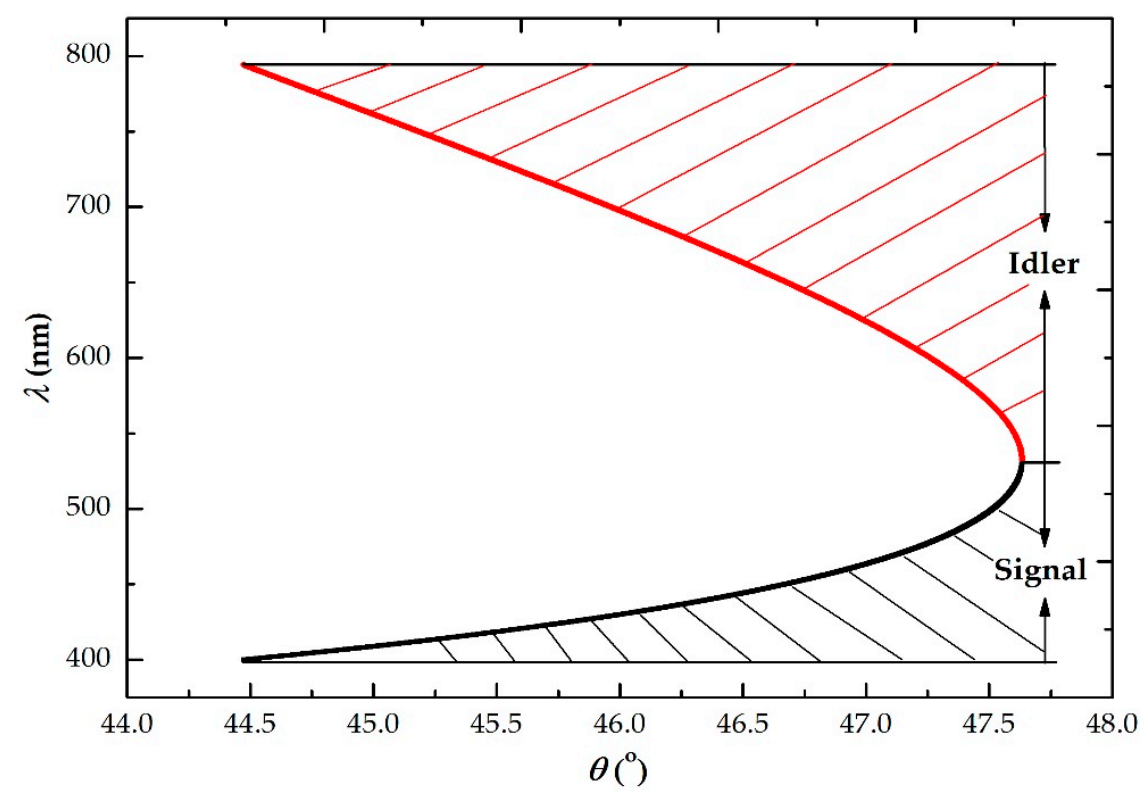

Figure 3. The curve of the wavelengths $\lambda$ of signal and idler changing with the change of phasematching angle $\theta$. The black curve in the lower part represents the wavelength of signal $\lambda_{s}(400 \mathrm{~nm}$ $\leq \lambda_{s} \leq 532 \mathrm{~nm}$ ), while the red curve in the upper part represents the wavelength of idler $\lambda_{i}(532 \mathrm{~nm}$ $\left.<\lambda_{i} \leq 794 \mathrm{~nm}\right)$.

In this part, assume that the three waves satisfy completely the phase matching conditions $\left(\theta=47.6339^{\circ}\right)$, which is $\Delta k=k_{s} \cos \alpha_{0}+k_{i} \cos \beta_{0}-k_{p}=0$, and the noncollinear angles of signal and idler are $\alpha_{0}$ and $\beta_{0}$, respectively. As the modification of correlated photon wavelengths, the corresponding non-collinear angle modifies as shown in Figure 4. It can be seen that the correlated photons of different wavelengths propagate along different non-collinear angles. When the wavelength of both ends approaches the degenerate wavelength at $532 \mathrm{~nm}$, the non-collinear angle decreases, and it is $0^{\circ}$ at the point of $532 \mathrm{~nm}$. This phenomenon conforms to the condition of complete phase matching.

In the actual process, if the pump beam in the crystal direction remains the same, the signal along the non-collinear angle $\left(\alpha_{0}+\Delta \alpha\right)$, corresponding idler will along $\left(\beta_{0}+\Delta \beta\right)$. At this point, the phase mismatch $\Delta k_{\text {act }}$ satisfies:

$$
\Delta k_{a c t}=k_{s} \cos \alpha+k_{i} \cos \beta-k_{p}
$$

where, $\alpha$ is the actual propagation direction of signal, which is the sum of non-collinear angle $\alpha_{0}$ that is completely phase matching and the non-collinear angular variation $\Delta \alpha$. Similarly, $\beta$ is the actual propagation direction of idler and it is the sum of non-collinear angle $\beta_{0}$ that is completely phase matching and the variation $\Delta \beta$. 


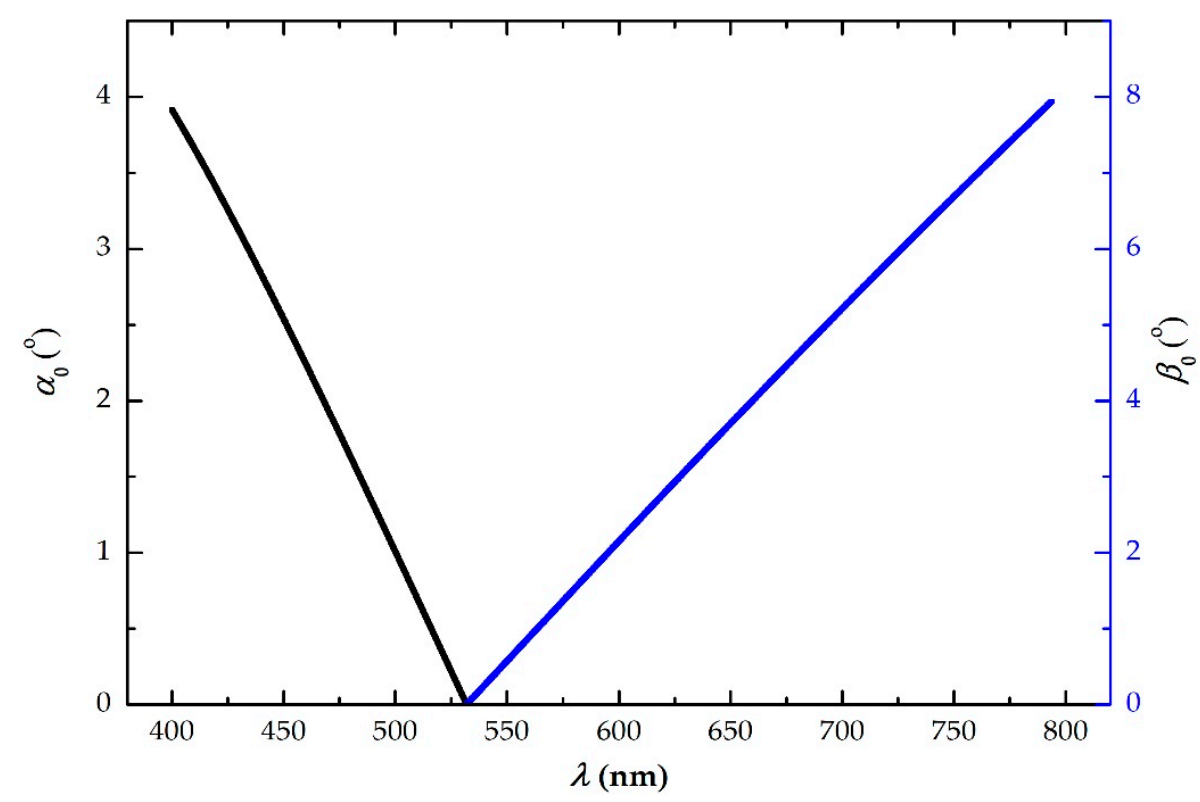

Figure 4. The relationship between the wavelength and the non-collinear angle of the correlated photon in the complete phase matching.

In order to research the variation of signal non-collinear angle, $\Delta k_{a c t}$ is expanded in a Taylor series:

$$
\Delta k_{a c t}=\Delta k+\frac{\partial \Delta k}{\partial \alpha} \cdot \Delta \alpha+\frac{1}{2 !} \frac{\partial^{2} \Delta k}{\partial \alpha^{2}} \cdot \Delta \alpha^{2}+\cdots
$$

The maximum phase mismatch is taken for $\Delta k_{a c t}$, and Equation (9) of $\Delta k_{a c t}$ expansion through second-order approximation can be rewritten as:

$$
\Delta k+\frac{\partial \Delta k}{\partial \alpha} \cdot \Delta \alpha+\frac{1}{2 !} \frac{\partial^{2} \Delta k}{\partial \alpha^{2}} \cdot \Delta \alpha^{2}+\frac{\pi}{L}=0
$$

with

$$
\left\{\begin{array}{l}
\frac{\partial \Delta k}{\partial \alpha}=-k_{s} \cdot \sin \alpha_{0}-k_{i} \cdot \sin \beta_{0} \cdot \frac{\partial \beta}{\partial \alpha} \\
\frac{\partial^{2} \Delta k}{\partial \alpha^{2}}=-k_{s} \cdot \cos \alpha_{0}-k_{i} \cdot \cos \beta_{0} \cdot\left(\frac{\partial \beta}{\partial \alpha}\right)^{2}-k_{i} \cdot \sin \beta_{0} \cdot \frac{\partial^{2} \beta}{\partial \alpha^{2}} \\
\frac{\partial \beta}{\partial \alpha}=\frac{n_{i} \lambda_{s}}{n_{s} \lambda_{i}} \cdot \frac{\cos \beta_{0}}{\cos \alpha_{0}} \\
\frac{\partial^{2} \beta}{\partial \alpha^{2}}=\frac{n_{i} \lambda_{s}}{n_{s} \lambda_{i}} \cdot \frac{-\sin \beta_{0}}{\cos \alpha_{0}} \cdot \frac{\partial \beta}{\partial \alpha}+\frac{n_{i} \lambda_{s}}{n_{s} \lambda_{i}} \cdot \cos \beta_{0} \cdot \frac{\sin \alpha_{0}}{\cos ^{2} \alpha_{0}}
\end{array}\right.
$$

Simplifying Equation (10), the variation of signal non-collinear angle $(\Delta \alpha)$ can be written as:

$$
\Delta \alpha=\frac{g_{s}-h_{s}}{w}
$$

with

$$
\left\{\begin{array}{l}
g_{s}=2 \pi \cdot\left[\frac{n_{s}}{\lambda_{s}} \cdot \sin \alpha_{0}+\left(\frac{n_{i}}{\lambda_{i}}\right)^{2} \cdot \frac{\lambda_{s}}{n_{s}} \cdot \frac{\sin \beta_{0} \cos \beta_{0}}{\cos \alpha_{0}}\right] \\
w=-2 \pi \cdot\left[\frac{n_{s}}{\lambda_{s}} \cdot \cos \alpha_{0}+\left(\frac{n_{i} \lambda_{s}}{n_{s} \lambda_{i}}\right)^{2} \cdot \frac{n_{i}}{\lambda_{i}} \cdot \frac{\cos 2 \beta_{0} \cos \beta_{0}}{\cos ^{2} \alpha_{0}}\right]+\frac{n_{i} \lambda_{s}}{n_{s} \lambda_{i}} \cdot \tan \alpha \cdot \frac{\cos \beta_{0}}{\cos \alpha_{0}} \\
f=2 w \cdot \frac{\pi}{L} \\
h_{s}=\sqrt{g_{s}^{2}-f}
\end{array}\right.
$$

In a similar way, the variation of idler non-collinear angle can be deduced which is similar to Equation (12). According to the above reasoning process, the variation of the non-collinear angle of the correlated photon is bound up with the non-collinear angle with complete phase matching and the action length of the three waves in the crystal. 


\section{Experimental Measurement}

In this part, an experimental platform is built to measure the properties of the angular distribution of the correlated photons generated by a BBO crystal, which verifies the theoretical research on the non-collinear angular variation through experimental method. The layout of the experimental measurement setup is shown in Figure 5.

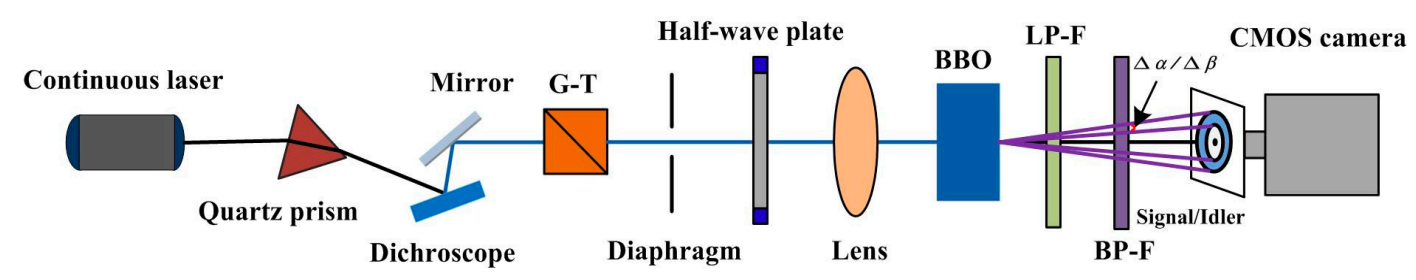

Figure 5. Experimental measurement setup.

The pump source is a continuous laser of $266 \mathrm{~nm}$ with the maximum power up to $55 \mathrm{~mW}$. The quartz prism (vertex angle of $70^{\circ}$ ) and the dichroscope (reflectivity of $98 \%$ in the wavelength of $266 \mathrm{~nm}$ ) eliminate the residual $532 \mathrm{~nm}$ in the pump beam of $266 \mathrm{~nm}$. Moreover, the G-T represents the Glan-Taylor polarizer made of $\alpha-\mathrm{BBO}$ and it is plated with $200 \mathrm{~nm}$ to $400 \mathrm{~nm}$ anti-reflection film, which modifies the polarization ratio of the pump, increases the proportion of e light in type-I SPDC, and further increases the correlated photon number generated. The zero-order half-wave plate is used to change the phase difference of $1 / 2$ wavelength to realize the opening and closing of the correlated photons generation process. A plano-convex lens with a focal distance of $150 \mathrm{~mm}$ focuses the pump beam at the entrance of the nonlinear crystal.

The nonlinear crystal used negative uniaxial $\mathrm{BBO}$ is $2 \mathrm{~mm}$ long, cut for type-I collinear phase-matching at $\theta=47.63^{\circ}$. Of course, the crystal phase-matching condition also can be turned to $n(\omega)=n_{p}(2 \omega)\left(n(\omega)\right.$ and $n_{p}(2 \omega)$ said the refractive index of the correlated photon and the pump) due to $k_{s, i}=n(\omega) \cdot \frac{\omega}{c}\left(k_{s, i}\right.$ and $\omega$ are, respectively, the wave number and frequency of the correlated photon, $c$ is the photon propagation speed in a vacuum). The results show that the phase-matching condition can be regarded as the refractive index of the correlated photon in the crystal is equivalent to that of the pump. In the case that most nonlinear optical crystals in the visible are in normal dispersion, and the higher the wave frequency, the greater the refractive index, namely $n(\omega)<n_{p}(2 \omega)$. Therefore, the phasematching condition cannot be satisfied theoretically when the wave beam propagates in isotropic medium. However, for the anisotropic BBO crystal, due to the birefringence effect, two waves with different refractive indices are allowed to propagate in the same direction, and the phase matching can be realized by the effect in the normal dispersion range.

The long-pass filter (with a transmittance of over $95 \%$ across the spectrum from $400 \mathrm{~nm}$ to $794 \mathrm{~nm}$ ) and the band-pass filter are represented as LP-F and BP-F, respectively, whose functions are to eliminate any residual radiation at the continuous laser and selecting the wavelength of the correlated photons. Moreover, the non-collinear angular variation and the divergence angular distribution are recorded by means of the imaging device of CMOS camera, whose effective area is $13.312 \mathrm{~mm} \times 13.312 \mathrm{~mm}$ and readout mode is chosen $4 \times 4$, placed in the behind of BP-F. Observe the spectral distribution of correlated photons emitted from the back of $\mathrm{BBO}$ crystal, and the results will be presented in Figure 6. It can be found that the signal and idler wavelength of $472 \mathrm{~nm}$ and $610 \mathrm{~nm}$ are a pair of correlated photons. It is observed that the same ring images appear as the theoretical analysis, in which the spot in each central same area is composed by $266 \mathrm{~nm}$ pump not eliminated completely. Although the detection sensitivity of CMOS camera in $400 \mathrm{~nm}$ to $794 \mathrm{~nm}$ is at least 6 times higher than $266 \mathrm{~nm}$, however, the correlated number is 8 orders of magnitude lower than the pump, the ring cannot be seen without the filters. The results of the theoretical calculations, experimental measurements, and detailed explanations will be developed in the next section. 


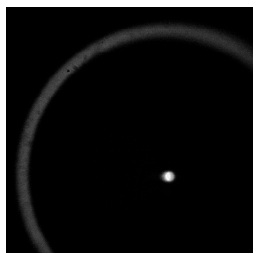

(a)

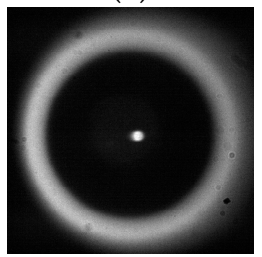

(e)

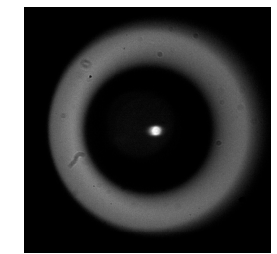

(b)

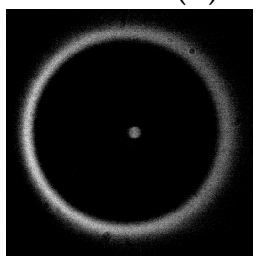

(f)

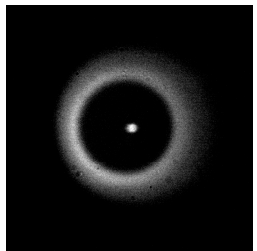

(c)

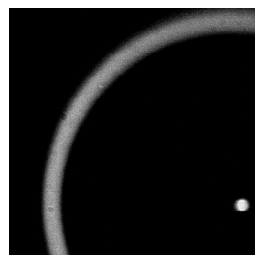

(g)

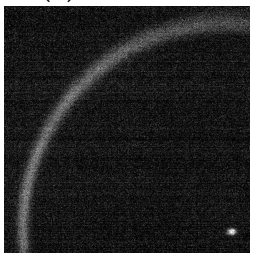

(h)



(d)

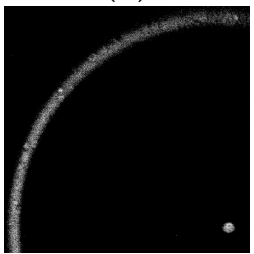

(i)

Figure 6. (a-i) Experimental results of the correlated photon rings with different wavelengths. (a) $\lambda=415 \mathrm{~nm}$, (b) $\lambda=472 \mathrm{~nm}$, (c) $\lambda=550 \mathrm{~nm},(\mathbf{d}) \lambda=572 \mathrm{~nm},(\mathbf{e}) \lambda=605 \mathrm{~nm},(\mathbf{f}) \lambda=610 \mathrm{~nm},(\mathrm{~g}) \lambda=685 \mathrm{~nm}$, (h) $\lambda=700 \mathrm{~nm}$, (i) $\lambda=710 \mathrm{~nm}$.

\section{Results Analysis}

According to theoretical analysis, the correlated photon ring width is related to the non-collinear angular variation, that is, the ring width at different wavelengths is different, and the corresponding non-collinear angular variation is also different. In this section, first using the Equation (12) to calculate the $415 \mathrm{~nm}, 472 \mathrm{~nm}, 550 \mathrm{~nm}, 572 \mathrm{~nm}, 605 \mathrm{~nm}$, $610 \mathrm{~nm}, 685 \mathrm{~nm}, 700 \mathrm{~nm}$, and $710 \mathrm{~nm}$, a total of nine wavelengths corresponding noncollinear angular variation, we find that the theoretical results have a floating range. Then, the ring profile measured by CMOS camera is processed, and the width values can be obtained with the image processing technology, finally converted into the non-collinear angular variation behind crystal (not exuded). Figure 7a shows the theoretical simulations and experimental measurements of the non-collinear angular variation at different correlated photon wavelengths. The black and red curves represent the theoretical values and the measured values of non-collinear angular variation, respectively. Figure $7 \mathrm{~b}$ illustrates the error bars of the experimental data. It can be observed that the measured values are less than the theoretical simulation, which is the result of the theoretical research under the premise of the largest phase mismatch. Also, it provides reference and guidance for predicting the maximum width and position of correlated photon rings. The non-collinear angular variations of $472 \mathrm{~nm}, 550 \mathrm{~nm}$ and $572 \mathrm{~nm}$ are simulated theoretically, higher than the other wavelengths, and the experimental results are consistent with the theory. Meanwhile, with the decrease of signal wavelength and the increase of idler wavelength, the difference between the theoretical and measured values of the non-collinear angular variation decreases. At the wavelengths of $685 \mathrm{~nm}$ and $550 \mathrm{~nm}$, the difference between theory and measured data are minimum $0.0168^{\circ}$ and maximum $0.1906^{\circ}$, respectively. The reason is that the closer the non-collinear angle is to the degenerate wavelength, the greater the influence of the experimental conditions such as phase-matching angle, pump incident angle, and diameter.

The location and size of the ring need to determine before calibrating the photodetector using correlated photons. To understand and determine the position and size of the ring before using correlated photons to carry out the photodetector calibration, another physical quantity, divergence angle, describing the spatial properties of the correlated photons, is introduced. It is the included angle between the outer circle of the correlated photon and the pump beam emitted from the back of the crystal, which is half of the apex angle of the spatial propagation cone, and in Figure 6, is expressed as $\theta_{S} / \theta_{i}$ representing respectively the divergence angle of signal or idler. The non-collinear angle is the between the propagation direction of the correlated photon in the crystal and the pump beam, while the divergence angle is another form of its existence outside the crystal. And the conversion between the two angles can be made by Snell's Law. Therefore, measured values of divergence angles 
at different wavelengths are obtained based on the correlated photon rings in the above experiment, however, the theoretical simulations require combining Equations (3)-(5) and Snell's Law, as presented in Figure 8. At both ends of the degenerate wavelength of $532 \mathrm{~nm}$ (415 $\mathrm{nm} \leq \lambda<532 \mathrm{~nm}$ and $550 \mathrm{~nm} \leq \lambda \leq 710 \mathrm{~nm}$ ), the divergence angle decreases as the wavelength of signal increases, while is diametrically opposite for the relationship between the idler wavelength and divergence angle, which is increases as the wavelength of idler increases. When the correlated photon wavelengths are $472 \mathrm{~nm}, 550 \mathrm{~nm}$ and $572 \mathrm{~nm}$ (near degeneracy), the measured average of the divergence angle differs by $1.73^{\circ}$ from the theoretical. On the one hand, it has an error of $0.25^{\circ}$ between the cutting angle and phase-matching angle which causes the degeneracy wavelength to shift. On the other hand, the effect of non-collinear angle on the phase-matching angle of degenerate collinear wavelength is significant. It can be seen from Figure 7 that the non-collinear angular variation mean of the three wavelengths is $0.0156^{\circ}$, which is greater than the others.
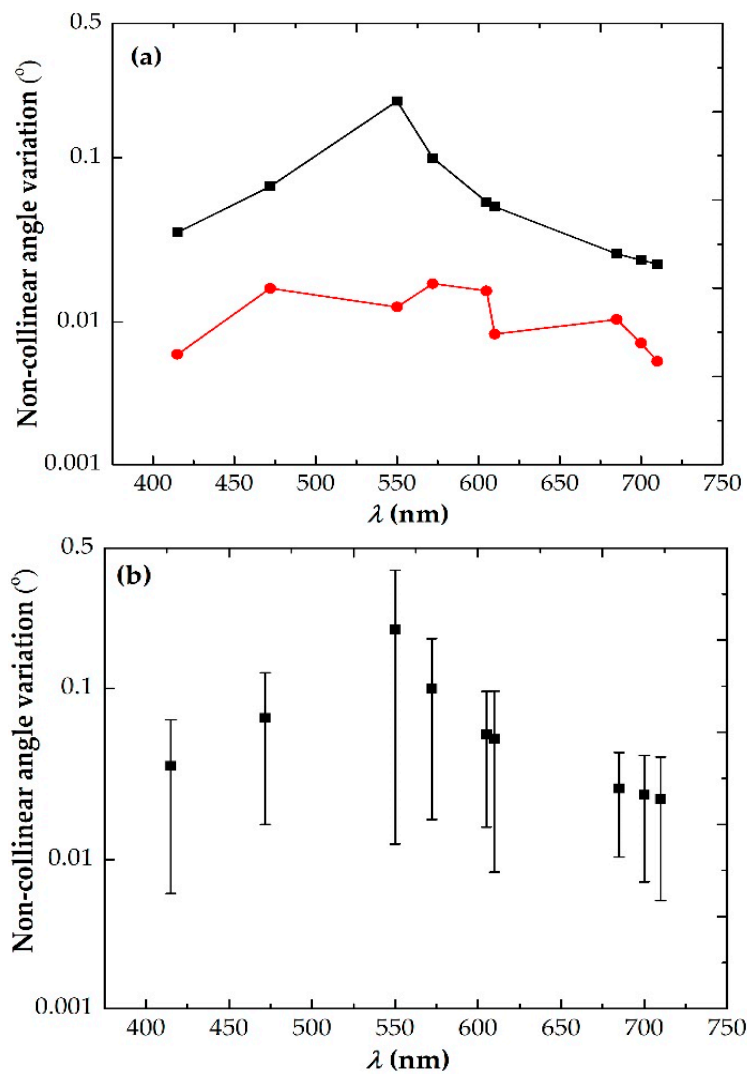

Figure 7. (a) Theoretical simulation and experimental measurement of the noncollinear angular variation at different photon wavelengths. The curves of black and red respectively represent the theoretical values and the measured values of non-collinear angular variation. (b) The error bars of the experimental data of the non-collinear angle variation. 

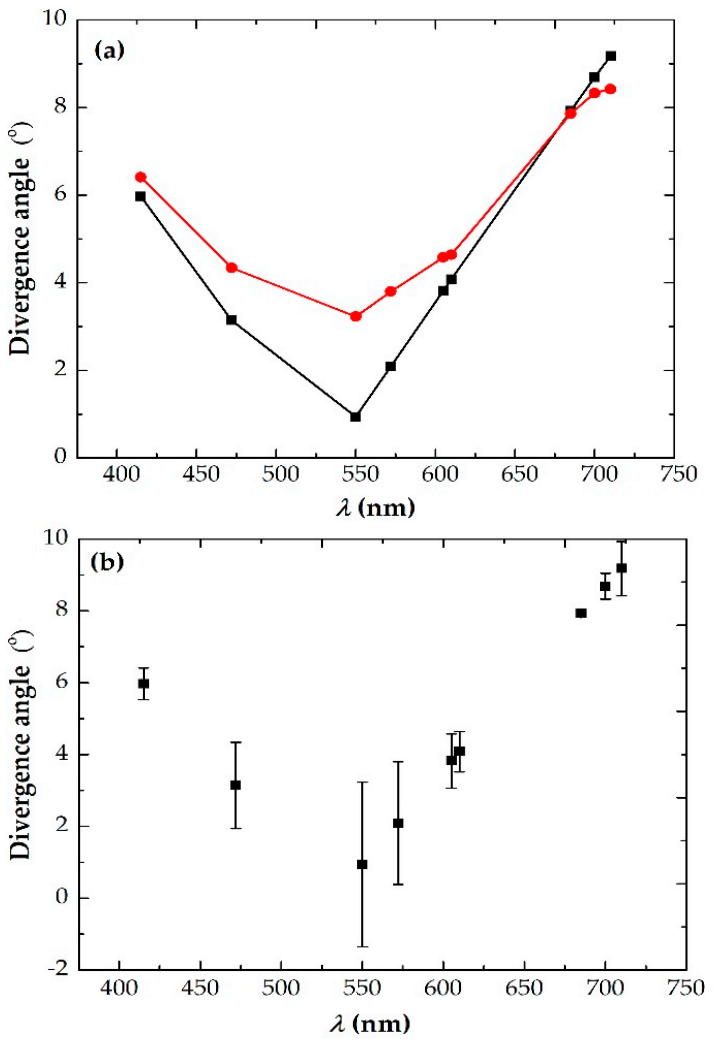

Figure 8. (a) Theoretical simulation and experimental measurement of the divergence angular spectrum at different photon wavelengths. The black curve represents the theoretical values, and the red curve is the experimental results. (b) The error bars of the experimental data of the divergence angle.

\section{Discussions}

The main result of this work is based on the quantity expression of phase-matching condition, and the theory studies the second-order change of the non-collinear angle with the wavelength of the correlated photons. At the same time, the parameters of the characterization spatial properties (the non-collinear angle of correlated photon, spectral width, divergence angle) are quantitatively described and analyzed without varying the parameters such as pump and crystal. In the experiment, a pair of correlated photon images can be observed and recorded, which verifies the process of producing the correlated photons in SPDC satisfying the law of energy conservation. However, the previous works mainly studied the spatial distribution of correlated photons produced by varying the pump parameters (such as, focusing on pump beam and changing pump spectral width) during the process of SPDC, which influenced the spatial symmetry of the two-photon field [25,40-42]. Also, a qualitative study was implemented for the spatial characteristics of correlated photons under the condition that the diameter, near field and far field of pump were considered [43]. Moreover, the spectral characteristics of the type-I SPDC, which were in the three commonly phase-matching conditions (collinear degenerate, noncollinear degenerate and collinear nondegenerate), were reported, and the relationship between spectroscopy and photon freedom entanglement was studied, which provided the theoretical guidance for the application of quantum ghost imaging [44], interference effect [45,46], and other techniques. The entangled two-photon field generated by SPDC phase matching in BBO crystal was explored in depth by Karan et al [47], who summarized the effects of various crystal and pump parameters on the entangled two-photon field, in order to illustrate how various experimental parameters affect the photon pairs generated. Finally, the two-photon wave function was derived based on angle-orbital angular momentum and the two-photon angular Schmidt spectrum was calculated. From the perspective of quantum electrodynamics, Forbes et al [48] quantitatively analyzed the degenerate down conversion mechanism of 
localized and nonlocalized, providing a reasonable explanation for the uncertainty of the position-momentum of the correlated photon propagation.

The spatial properties of the SPDC in type-I BBO uniaxial crystal with degenerate wavelength collinear phase-matching are investigated theoretically and experimentally. Based on the quantity expression of phase-matching condition, the relationship between the non-collinear angle and the correlated photon wavelength has been presented. Also, the theoretical process of the non-collinear angular variation is derived and analyzed, and the results have a floating range of $0.0235^{\circ}-0.2032^{\circ}$. Then, the spatial divergence angle coming out of the crystal, is calculated. Of course, the correlated photon rings are measured by CMOS cameras and the measured image can be converted to the corresponding measurements of non-collinear angular variations and divergence angles. The experimental measurement results verify the correctness of the numerical simulation of non-collinear angular variation in the range. As for the divergence angle, the measurements at both ends of the degenerate wavelength $532 \mathrm{~nm}$ (except for three nearby wavelengths) are consistent with the theoretical simulation. And the non-collinear angular variation mean of three correlated photon wavelengths near the degenerate wavelength is larger than others, which also has a great influence on the phase-matching angle of the degenerate wavelength collinear. Moreover, at the phase-matching angle $47.63^{\circ}$, the measured divergence angle decreases with the increase of signal and increases with the increase of idler, and the result is agreement with theoretical analysis. The theoretical analysis model of the spatial properties of the correlated photon pairs established in the paper will lay a foundation for a deeper understanding of the positions and characteristics of parametric photons in advance in the photodetector calibration process.

Author Contributions: Conceptualization, X.Z. and L.Y.; data curation, L.Y. and Y.H.; formal analysis, J.L., W.Z., M.X., and Y.H.; investigation, J.L., W.Z., and Y.H.; methodology, L.Y.; validation, L.Y.; Writing-original draft, L.Y.; Writing-review \& editing, X.Z. and L.Y. All authors have read and agreed to the published version of the manuscript.

Funding: This research and the APC were funded by Chinese National Key Research and Development project under grant No. 2018YFB0504602 and the National Natural Science Foundation of China under grant No. 41805019, National Defense Technology Foundation program (No. JSJL2018210C003).

Data Availability Statement: Data available on request due to restrictions eg privacy or ethical. The data presented in this study are available on request from the corresponding author. The data are not publicly available due to the Chinese National Key Research and Development project not been completed and some of the data involved in this article are still in the confidential stage.

Conflicts of Interest: The authors declare no conflict of interest.

\section{References}

1. Byer, R.L.; Harris, S.E. Power and Bandwidth of Spontaneous Parametric Emission. Phys. Rev. 1968, 168, 1064-1068. [CrossRef]

2. Burnham, D.C.; Weinberg, D.L. Observation of Simultaneity in Parametric Production of Optical Photon Pairs. Phys. Rev. Lett. 1970, 25, 84-87. [CrossRef]

3. Dayan, B.; Pe'Er, A.; Friesem, A.A.; Silberberg, Y. Two Photon Absorption and Coherent Control with Broadband Down-Converted Light. Phys. Rev. Lett. 2004, 93, 023005. [CrossRef]

4. Mandel, L.; Wolf, E. Optical Coherence and Quantum Optics. In Optical Coherence and Quantum Optics; Cambridge University Press: Cambridge, UK, 1995; Volume 378, p. 454. [CrossRef]

5. Horodecki, R.; Horodecki, P.; Horodecki, M.; Horodecki, K. Quantum entanglement. Rev. Mod. Phys. 2009, 81, 865-942. [CrossRef]

6. Fox, M. Quantum Mechanics: Theory and Experiment, by Mark Beck. Contemp. Phys. 2013, 54, 77. [CrossRef]

7. Fox, M.; Javanainen, J. Quantum Optics: An introduction. Phys. Today 2007, 60, 74-75.

8. Klyshko, D.N. Coherent photon decay in a nonlinear medium. Pis'ma Zh. Eksp. Teor. Fiz. 1967, $23,490$.

9. Dong, M.-X.; Zhang, W.; Shi, S.; Wang, K.; Zhou, Z.-Y.; Liu, S.-L.; Ding, D. Two-color hyper-entangled photon pairs generation in a cold $\wedge 85 \mathrm{Rb}$ atomic ensemble. Opt. Express 2017, 25, 10145. [CrossRef]

10. Gisin, N.; Ribordy, G.; Tittel, W.; Zbinden, H. Quantum cryptography. Rev. Mod. Phys. 2002, 74, 145-195. [CrossRef]

11. Cheung, J.Y.; Chunnilall, C.J.; Woolliams, E.R.; Fox, N.P.; Mountford, J.R.; Wang, J.; Thomas, P.J. The quantum candela: A redefinition of the standard units for optical radiation. J. Mod. Opt. 2007, 54, 373-396. [CrossRef] 
12. Rarity, J.G.; Tapster, P.R. Experimental Violation of Bell's Inequlity Based on Phase and Momentum. Phys. Rev. Lett. 1990, 64, 2465-2498. [CrossRef]

13. Kwiat, P.G.; Steinberg, A.M.; Chiao, R.Y. High-visibility interference in a Bell-inequality experiment for energy and time. Phys. Rev. A 1993, 47, R2472-R2475. [CrossRef] [PubMed]

14. Valencia, A.; Scarcelli, G.; Shih, Y. Distant clock synchronization using entangled photon pairs. Appl. Phys. Lett. 2004, 85, 2655-2657. [CrossRef]

15. Jennewein, T.; Simon, C.; Weihs, G.; Weinfurter, D.H.; Zeilinger, A. Quantum Cryptography with Entangled Photons. Phys. Rev. Lett. 2000, 84, 4729-4732. [CrossRef] [PubMed]

16. Tittel, W.; Brendel, J.; Zbinden, H.; Gisin, N. Quantum Cryptography Using Entangled Photons in Energy-Time Bell States. Phys. Rev. Lett. 2000, 84, 4737-4740. [CrossRef] [PubMed]

17. Matthews, J.C.F.; Zhou, X.-Q.; Cable, H.; Shadbolt, P.J.; Saunders, D.J.; Durkin, G.A.; Pryde, G.J.; O’Brien, J.L. Towards practical quantum metrology with photon counting. Npj Quantum Inf. 2016, 2, 16023. [CrossRef]

18. Migdall, A.; Datla, R.; Sergienko, A.; Orszak, J.S.; Shih, Y.H. Measuring absolute infrared spectral radiance with correlated visible photons: Technique verification and measurement uncertainty. Appl. Opt. 1998, 37, 3455-3463. [CrossRef]

19. Polyakov, S.V.; Migdall, A.L. Quantum radiometry. J. Mod. Opt. 2009, 56, 1045-1052. [CrossRef]

20. Cheung, J.Y.; Chunnilall, C.J.; Porrovecchio, G.; Smid, M.; Theocharous, E. Low optical power reference detector implemented in the validation of two independent techniques for calibrating photon-counting detectors. Opt. Express 2011, 19, 20347-20363. [CrossRef]

21. Lemieux, S.; Giese, E.; Fickler, R.; Chekhova, M.V.; Boyd, R.W. A primary radiation standard based on quantum nonlinear optics. Nat. Phys. 2019, 15, 529-532. [CrossRef]

22. Hu, Y.; Li, J.; Gao, D.; Zheng, X. Absolute radiance measurement based on correlated photons calibration. Eur. Phys. J. D 2020, 74, 8. [CrossRef]

23. Prutchi, D.; Prutchi, S.R. Exploring Quantum Physics through Hands-On Projects. In Exploring Quantum Physics Through Hands-On Projects; John Wiley \& Sons, Inc.: Hoboken, NJ, USA, 2012; Volume 10, pp. 203-243. [CrossRef]

24. Rubin, M.H.; Klyshko, D.N.; Shih, Y.H.; Sergienko, A.V. Theory of two-photon entanglement in type-II optical parametric down-conversion. Phys. Rev. A 1994, 50, 5122-5133. [CrossRef] [PubMed]

25. Joobeur, A.; Saleh, B.E.A.; Teich, M.C. Spatiotemporal coherence properties of entangled light beams generated by parametric down-conversion. Phys. Rev. A 1994, 50, 3349-3361. [CrossRef] [PubMed]

26. Joobeur, A.; Saleh, B.E.A.; Larchuk, T.S.; Teich, M.C. Coherence properties of entangled light beams generated by parametric down-conversion: Theory and experiment. Phys. Rev. A 1996, 53, 4360-4371. [CrossRef]

27. Molina-Terriza, G.; Minardi, S.; Deyanova, Y.; Osorio, C.I.; Hendrych, M.; Torres, J.P. Control of the shape of the spatial mode function of photons generated in noncollinear spontaneous parametric down-conversion. Phys. Rev. A 2005, 72, 913-922. [CrossRef]

28. Torres, J.P.; Molina-Terriza, G.; Torner, L. The spatial shape of entangled photon states generated in non-collinear, walking parametric downconversion. J. Opt. B Quantum Semiclass. Opt. 2005, 7, 235-239. [CrossRef]

29. Kumbhakar, P.; Kobayashi, T. Ultrabroad-band phase matching in two recently grown nonlinear optical crystals for the generation of tunable ultrafast laser radiation by type-I noncollinear optical parametric amplification. J. Appl. Phys. 2003, 94, 1329. [CrossRef]

30. Devaux, F.; Lantz, E. Spatial and temporal properties of parametric fluorescence around degeneracy in a type I LBO crystal. Eur. Phys. J. D 2000, 8, 117-124. [CrossRef]

31. Liu, H.; Wei, Z.; Guofu, C.; Yishan, W.; Zhao, C.; Chi, R. Investigation of spectral bandwidth of optical parametric amplification. Appl. Phys. A 2004, 79, 569-576. [CrossRef]

32. Shirakawa, A.; Kobayashi, T. Noncollinear phase and group velocity matching of optical parametric amplifier for ultrashort pulse generation. IEICE Trans. Electron. 1998, E81C, 246-253.

33. Shirakawa, A.; Kobayashi, T. Nncollinearly phase-matched femtosecond optical parametric amplification with a $2000 \mathrm{~cm}^{-1}$ bandwidth. Appl. Phys. Lett. 1998, 72, 147-149. [CrossRef]

34. Surhone, L.M.; Timpledon, M.T.; Marseken, S.F. Snell's Law; Betascript Publishing: Beau Bassin, Mauritius, 2010.

35. Hong, C.K.; Mandel, L. Theory of parametric frequency down conversion of light. Phys. Rev. A 1985, 31, 2409-2418. [CrossRef] [PubMed]

36. Klyshko, D.N. Photons Nonlinear Optics; CRC Press: Boca Raton, FL, USA, 1988

37. Kato, K. Second-harmonic generation to $2048 \AA$ in $\beta$-BaB2O4. IEEE J. Quantum Electron 1986, 22, 1013-1014. [CrossRef]

38. Wu, S.; Blake, G.A.; Sun, S.; Ling, J. A multicrystal harmonic generator that compensates for thermally induced phase mismatch. Opt. Commun. 2000, 173, 371-376. [CrossRef]

39. Barnes, N.P.; Corcoran, V.J. Parametric generation processes: Spectral bandwidth and acceptance angles. Appl. Opt. 1976, 15, 696-699. [CrossRef]

40. Grice, W.P.; Bennink, R.S.; Zhao, Z.; Meyer, K.; Shaw, R. Spectral and spatial effects in spontaneous parametric down-conversion with a focused pump. In Quantum Communications and Quantum Imaging VI, Proceedings of the SPIE Optical Engineering + Applications, San Diego, CA, USA, 10-14 August 2008; Ronald, E.M., Yanhua, S., Keiths, D., Eds.; SPIE: San Diego, CA, USA, 2008; Volume 7092, p. 70920Q. 
41. Grice, W.P.; Walmsley, I.A. Spectral information and distinguishability in type-II down-conversion with a broadband pump. Phys. Rev. A 1997, 56, 1627-1634. [CrossRef]

42. Bennink, R.S.; Liu, Y.; Earl, D.D.; Grice, W. Spatial distinguishability of photons produced by spontaneous parametric downconversion with a focused pump. Phys. Rev. A 2006, 74, 023802. [CrossRef]

43. Beržanskis, A.; Chinaglia, W.; Lugiato, L.; Feller, K.-H.; Di Trapani, P. Spatial structures in optical parametric amplification. Phys. Rev. A 1999, 60, 1626-1635. [CrossRef]

44. Moreau, P.-A.; Toninelli, E.; Morris, P.A.; Aspden, R.S.; Gregory, T.; Spalding, G.; Boyd, R.W.; Padgett, M.J. Resolution limits of quantum ghost imaging. Opt. Express 2018, 26, 7528-7536. [CrossRef]

45. Baek, S.Y.; Kim, Y.H. Spectral properties of entangled-photons generated via type-I spontaneous parametric downconversion. In Proceedings of the Conference on Lasers and Electro-Optics (CLEO), Baltimore, MD, USA, 6-11 May 2007; ISBN 978-1-55752834-6.

46. Walborn, S.; Monken, C.; Pádua, S.; Ribeiro, P.H.S. Spatial correlations in parametric down-conversion. Phys. Rep. 2010, 495, 87-139. [CrossRef]

47. Karan, S.; Aarav, S.; Bharadhwaj, H.; Taneja, L.; De, A.; Kulkarni, G.; Meher, N.; Jha, A.K. Phase matching in $\beta$-barium borate crystals for spontaneous parametric down-conversion. J. Opt. 2020, 22, 083501. [CrossRef]

48. Forbes, K.A.; Ford, J.S.; Jones, G.A.; Andrews, D.L. Quantum delocalization in photon-pair generation. Phys. Rev. A 2017, 96, 023850. [CrossRef] 